\title{
FISIOTERAPIA
}

\section{Efectos de un programa de ejercicio basado en boxeo sobre el balance en un sujeto con Enfermedad de Parkinson: estudio de caso}

\author{
Ingrid Vanessa Suarez González , Fabiola González Ortiz
}

\begin{abstract}
Resumen
Introducción: La Enfermedad de Parkinson (EP) es una enfermedad que genera alteraciones primarias en algunas funciones y estructuras del sistema nervioso central. Por sus características degenerativas y multisistémicas se manifiesta a través de sintomatología motora, cognitiva y autonómica, como bradicinesia, rigidez, temblor, déficit en funciones ejecutivas, alteraciones del habla, exceso de sudoración y micción entre otras, que genera impacto en la participación y aumentando el riesgo de morbilidad como las que derivan de las caídas a causa de la inestabilidad postural y alteracion en el balance. El tratamiento de EP tiene como objetivo reducir y controlar la progresión de la sintomatología debido que aún no tiene cura, está basado principalmente en la prescripción farmacológica, sin embargo, complementario a ello a través de la última década se han implementado estrategias de intervención multidisciplinar, en la cuales alternativas efectuadas por los fisioterapeutas quienes diseñan programas de ejercicio físico de forma metódica y sistemática han demostrado ser beneficiosos dentro de los procesos de rehabilitación, para demostrar ello, han utilizado diferentes estrategias de entrenamiento y prescripción tales como cicloergometro, banda caminadora, actividades acuáticas, entre otras. Debido al impacto positivo en respuesta a intervenciones derivadas del ejercicio físico, los investigadores han continuado en la búsqueda de alternativas efectivas primordialmente desde estrategias multisensoriales e integradoras como el baile, la realidad virtual y el boxeo. Es por ello que surge la necesidad de generar evidencias que procedan de estas alternativas, favoreciendo aspectos propios de la estabilidad postural, la marcha y el balance, siendo esto trasferible a una mejor funcionalidad e independencia de los sujetos con EP.
\end{abstract}

Objetivo: Identificar los efectos generados por un programa de ejercicio basado en boxeo sobre el balance en un paciente con EP.

Material y Método: Se realizó un estudio cuasi experimental de caso único,

1. Fundación Escuela Colombiana de Rehabilitación, Colombia.

E-mail: ingridsuarez@ecr.edu.co

DOI: 10.26885/rcei.foro.2019.227

Trabajo publicado en acceso abierto bajo Licencia Creative Commons. 
efectuado a un Paciente de 50 años con EP en estadio inicial según Hoehn y Yahr, tipo rígido acinético, a quien se le realizó una intervención desde el ejercicio físico basado en Boxeo compuesto por 36 sesiones efectuadas durante 12 semanas. Se estableció como línea base la valoración del balance mediante Test Up and Go y Escala de Berg, revaluados posteriormente a la terminación del programa de ejercicio.

Resultados: En cuanto a la Escala de Berg el pretest revelo un puntaje de 48/56 con una clasificación de "riesgo de caída leve", en el postest se encontró un puntaje de 56/56 (semana 12), clasificado como sin riesgo. En cuanto al Test de Up and Go en la valoración inicial tuvo 8.27 segundos al efectuar la prueba, en las semanas 12 la prueba fue desempeñada en 6.32 segundos.

Conclusiones: Las personas con EP presentan alteración en la postura y marcha, lo que altera las habilidades para mantener el balance. Estrategias derivadas de la realización de ejercicio físico a través del entrenamiento del Boxeo, pueden disminuir de forma significativa el riesgo de caídas, manifestándose en mejores hallazgos en las pruebas relacionadas con la medición del balance, favoreciendo así la independencia funcional de los sujetos con esta condición de salud.

Palabras clave: enfermedad de Parkinson, boxeo, balance postural.

\section{Referencias}

Borrione, P., Tranchita, E., Sansone, P., \& Parisi, A. (2014). Effects of physical activity in Parkinson's disease: A new tool for rehabilitation. World Journal of Methodology, 4(3). 133-143. https://www.ncbi.nlm.nih.gov/ pmc/articles/PMC4202452/

Combs, S. et al. (2011). Boxing Training for Patients With Parkinson Disease: A Case Series. Rev Physical Therapy, 91(1) 132-142. https://academic. oup.com/ptj/article/91/1/132/2735142

Hurtado, F., Cárdenas, M., Cárdenas, F., León L. (2016), La enfermedad de Parkinson: Etiología, Tratamientos y Factores Preventivos. Rev Universitas Psychologica, 15(5).

Jankovic, J., \& Stacy, M. (2007), Medical Management of LevodopaAssociated Motor Complications in Patients with Parkinson's Disease. CNS Drugs, 21, 677-692. http://dx.doi. org/10.2165/00023210-200721080-00005

Tanaka, K., et al. (2009) Benefits of physical exercise on executive functions in older people with Parkinson's Disease. Brain Cong., 69, 435-441. 10.1016/j.bandc.2008.09.008 